\title{
Cronobiologia e Epilepsias
}

\author{
Sueli Rizzutti* \\ Mauro Muszkat** \\ Carlos José Reis de Campos***
}

\begin{abstract}
RESUMO
A cronobiologia é o estudo đos ritmos biológicos e de seus mecanismos subjacentes. A cronobiologia medica, em particular, tem interesse com o ritmo circadiano. O momento exato e as circunstáncias em que ocorre a crise epiléptica, como também a recorrencia de crises, sảo motivos de apreensảo para o paciente e para o neurologista. Em alguns pacientes, as crises epilépticas têrn uma recorrencia regular; em outros, há um quadro periódico sem crise e, apos alguns dias, surge um surto de crises epilépticas. Este trabalho discute a cronobiologia médica e sua aplicaçåo nas epilepsias.
\end{abstract}

\section{UNITERMOS}

Cronobiologia, epilepsia, ritmos circadianos.
Médica Neuropediatra, Mestre em Neuro. logia pela Escola Paulista de Medicina UNIFESP.

* Médico Neurologista, Doutor em Neurologia pela Escola Paulista de Medicina - UNIFESP.

*\#* Professor Adjunto e Chefe do Setor de Investigaçăo e Tratamento das Epilepsias (SITE) da Escola Paulista de Medicina UNIFESP.
Em neurociência, tem-se preocupado muito com o estudo do que se convencionou chamar de "organização temporal dos seres vivos". Os seres vivos estão organizados em todas as dimensões físicas, inclusive no espaço, no tempo e no processo evolutivo. Os seres vivos adaptaram-se não só à organização espacial do meio ambiente (adaptações anatômicas, bioquímicas), mas também à organização temporal desse meio ambiente. Pode-se estabelecer dois tipos de organização temporal no meio ambiente: a primeira estrutura temporal é composta por sequiência de eventos únicos, não-recorrentes; e a segunda tem sua estrutura composta por seqüências de eventos recorrentes.

O estudo dos ritmos biológicos e de seus mecanismos subjacentes denomina-se "cronobiologia". A estrutura temporal biológica, que possibilitou sua adaptação a fatores recorrentes ambientais, implica em flutuações periódicas dos diversos eventos biológicos. A essa variação sistemática, regular e periódica, dá-se o nome de "ritmicidade biológica". Os ritmos biológicos podem ser classificados em três grandes grupos, dependendo da frequêencia ou do período de recorrência do evento considerado:

- Ritmos circadianos: aqueles cujas flutuações completam-se a cada 24 horas, aproximadamente ( $24 \pm 4$ horas).

- Ritmos ultradianos: aqueles que apresentam mais de um ciclo completo a cada 24 horas (período menor que 20 horas).

- Ritmos infradianos: aqueles com período de repetição > 28 horas.

Mistlberger e Rusak ${ }^{1}$ referem que os ritmos diários, em uma grande variedade de espécies, incluindo os seres bumanos, mostram-se duradouros, desde que sob condições ambientais constantes. A persistência de ritmos diários constantes sugere que eles são gerados internamente pelo organismo e não são passivos de respostas aos estímulos ambientais.

As estruturas capazes de gerar os vários períodos dos diversos ritmos observados são chamados osciladores endógenos, marcapassos ou relógios biológicos ${ }^{2,3}$.

Os osciladores endógenos têm a propriedade de ser sincronizados (ou arrastados) por fatores cíclicos ambientais. Esses fatores recorrentes ambientais que podem regular o período e a fase dos osciladores endógenos 
são chamados de agentes sincronizadores, agentes arrastadores ou zeitgebers.

O principal sincronizador ambiental dos ritmos circadianos, para a maioria dos seres vivos, é a alternância entre claro-escuro do dia e da noite. No caso dos seres humanos, em particular, além do ciclo iluminação, fatores cíclicos decorrentes da organização social do trabalho e do lazer agem como poderosos zeitgebers ${ }^{1,2,3}$.

Os ritmos circadianos são relevantes no processo de adaptação dos organismos a seu meio ambiente. Importantes processos ocorrem nos mamíferos para que o meio interno adapte-se ao meio externo. $O$ conceito clássico de homeostase refere-se à integração de sistemas fisiológicos que tendem a uma estabilização (steadystate) das funções celulares e bioquímicas. A homeostase é um princípio essencial na organização dos sistemas biológicos e permite variações recorrentes, às vezes rítmicas. Os ritmos de maior complexidade são observados nos sistemas nervoso, circulatório e respiratório e são caracterizados por grande variabilidade individual.

Uma grande variedade de ritmos circadianos tem sido observada no homem, tais como a produção dos constituintes do sangue (crase sanguínea), funções fisiológicas, como temperatura corpórea, pulso, pressão arterial, incidência da fase do movimento rápido dos olhos, e padrões de comportamento ${ }^{4.5}$.

Encontramos, comumente, um padrão circadiano e um padrão sazonal na sintomatologia de doenças crônicas como rinite alérgica, angina, artrite reumatóide, osteoartrite, asma, epilepsia, hipertensão arterial e úlcera ${ }^{5}$. Autores como Smonlenski e D'Alonzo ${ }^{5}$ reforçam que o objetivo maior da cronobiologia médica está na cronofarmacologia (estudo dos ritmos biológicos e das medicações), levando em consideração o ritmo da cinética e da dinâmica das medicações com a manifestação rítmica da doença.

Lothman $^{6}$ refere que as características cronobiologicas da epilepsia são pouco compreendidas e muito intrigantes. Nesse caso, o principal objetivo da cronobiologia é tentar responder duas grandes questões. Por que as crises epilépticas ocorrem de modo aleatório em alguns pacientes e com um padrão de distribuição bem definido em outros? Por que as crises epilépticas ocorrem freqüentemente como eventos separados e ocasionalmente juntas, como no caso do status epilepticus?

Nota-se uma grande heterogeneidade quanto à frequiência de crises, encontrando-se, também, ciclos mensais com uma freqüência aleatória. $O$ momento de recorrência das crises sempre tem despertado grande interesse, originando estudos de avaliação sobre a predisposição de alguns pacientes terem suas crises em determinadas horas do dia $^{7,8}$.

No final da década de 20 e início da de 30 , alguns autores já monstravam interesse pelo momento da recorrência das crises, verificando a predisposição de alguns pacientes terem suas crises em determinadas horas do dia ${ }^{7,8}$. Embora pouco se conheça sobre o momento da recorrência de crises epilépticas ${ }^{9}$, alguns autores procuraram relacionar o estresse e a recorrência de crises $^{10}$, sendo propostos modelos matemáticos para explicar e predizer o momento de recorrência de novas crises $^{\prime \prime}$.

Stevens et al. ${ }^{12}$ analisaram 20 exames, com duração entre 17 e 72 horas, obtidos com telemetria em 5 pacientes com epilepsia, observaram que a crise epiléptica ocorria sempre em um intervalo de 90 a 100 minutos ou múltiplos desses intervalos e concluíram que a taxa de atividade epileptiforme interictal tende a flutuar em torno de uma média, variável para cada paciente. Kellaway et al. ${ }^{13}$ notaram ocorrência cíclica dos complexos ponta-onda em pacientes com epilepsia generalizada.

Lange et al. ${ }^{14}$ encontraram mudanças mínimas da atividade epileptiforme nos minutos que precedem as crises epilépticas.

Poucos autores investigaram ritmos circadianos endógenos ou ritmos ultradianos modulando o fenômeno epiléptico independentemente do sono, vigília ou do ciclo REM-REM ${ }^{13,15}$.

A monitorização prolongada tem mostrado que a atividade epileptiforme interictal não se distribui aleatoriamente e que tende a se modular de uma maneira que sugere a interação de influências ultradianas e circadianas ${ }^{13,16}$.

Bowman et al. ${ }^{17}$ estudaram a periodicidade das crises parciais complexas em nove pacientes e mostraram que as crises ocorriam de modo agrupado e obedeciam a um ritmo circadiano ( 24 horas) para alguns pacientes e circavigintiano para outros (a cada 21 dias).

Segundo Martins da Silva et al..$^{15}$, não há evidências de que ritmos circadianos afetem as atividades epilépticas interictais; contudo, a marcada periodicidade vista em alguns pacientes, mesmo em vigília, sugere ritmicidade ultradiana. A flutuação da atividade epiléptica interictal com aumento noturno pode refletir ritmos endógenos. Esses autores verificaram também que o padrão noturno da atividade epiléptica refletia ritmos endógenos circadianos ou ultradianos provavelmente idênticos àqueles que determinam os ciclos sono-vigília e REM-REM. 
Poirel ${ }^{18}$ sugere que a alta susceptibilidade para crises generalizadas tônico-clônicas corresponde a um baixo nível de atenção. Esse autor refere que, conhecendo os fatores temporais para a eventual ocorrência de uma crise epiléptica, se pode programar algumas estratégias para ajudar o paciente e também conclui que a cronobiologia permite o desenvolvimento de novos conceitos heurísticos no campo de neurociências.

Milton et al. ${ }^{9}$ realizaram um estudo prospectivo de 24 pacientes com a utilização de um diário com dados referentes às datas de ocorrência de crises epilépticas, durante cerca de 8 meses consecutivos, e concluíram que foi aleatória a ocorrência de crises cíclicas e/ou agrupadas (definidas como um aumento do número de dias com duas ou mais crises e/ou um aumento no número de intervalos curtos entre as crises). Balish et al. ${ }^{20}$ avaliaram também, com auxílio de calendários, a freqüência de crises de pacientes com epilepsia parcial, detectando tanto um padrão aleatório como ciclos mensais de recorrência.

Gotman e Koffler ${ }^{19}$ observaram uma grande correlação entre regiões mostrando atividade epileptiforme interictal e as regiōes nas quais as crises epilépticas se iniciam, não encontrando correlação entre a atividade epileptiforme e o nível sérico das drogas antiepilépticas. Concluíram que a flutuação da atividade epileptiforme interictal é decorrente das próprias crises epilépticas.

Pacientes na faixa etária entre 5 e 10 anos, com crises de ausência típica, foram avaliados com videoeletrencefalografia por telemetria, sendo observadas manifestações eletroclínicas mais freqüentes pela manhã em comparação com o período da tarde, e mais à tarde em comparação com o sono. Foi observado também que os componentes lentos dos complexos ponta-onda foram mais deformados e menos freqüentes durante o sono $^{21.22}$.

Horita et al. ${ }^{22}$ avaliaram quatro crianças que apresentavam crises de ausência típica, com monitorização ambulatorial eletrencefalográfica, utilizando gravador em fitas cassete, em registros com duração de 22 horas, e não encontraram maior freqüência dos complexos ponta-onda durante a vigília.

Ellis $^{23}$ refere que pesquisas cronobiologicas usando a atividade epileptiforme ictal apresentam algumas dificuldades, como: pacientes com epilepsia podem ter eventos ictais sem nenhuma manifestação clínica detectada por observadores; os pacientes podem, em muitos episódios, não lembrar das manifestações epilépticas ocorridas; e também a manifestação epiléptica pode ocorrer durante a noite e não ser detectada.

Existe uma questão importante sobre a aplicação dos princípios cronobiológicos em epilepsia, focalizando principalmente o momento preciso em que ocorre a crise epiléptica. É de fundamental importância saber qual é o momento crítico em que ocorre a crise epiléptica, pois a habilidade em prever esse momento facilitaria muito os cuidados para com o paciente epiléptico. Embora prever a ocorrência de eventos, conhecendo a sua periodicidade de acordo com mudanças cíclicas, tenha um grande interesse na prática médica diária, o estudo cronobiológico das crises epilépticas ainda precisa ser ampliado e consolidado, levando-se em conta, especialmente, o fato de as epilepsias serem constituídas por grupo heterogêneo de crise com mecanismos fisiopatológicos distintos.

\section{SUMMARY}

Chronobiology and epilepsies

Chronobiology is the study of biologic rhythms and biologic time structure. and other bioperiogy, in particular, is concerned with circadian (24-hs) and other bioperiodic influences on human diseases. The timing and circumstances of seizure recurrence are a source of apprehension for the patient and a mystery for the neurologist. In some patients, seizures appear to recur regularly. In others, there are fairly long seizure-free intervals and then, within a few days, there is a "burst" of seizure activity, referred to as clustering. Some important papers from medical chronobiology have been chosen for their relevance to epilepsy, although we need more studies applied to epilepsies, due to its syndromic nature and heterogenic physiopathology.

\section{KEYWORDS}

Chronobiology, epilepsy, circadian rhytms.

\section{Referências}

1. Mistlberger R \& Rusak B. Mechanisms and Models of the Circadian Timekeeping System. In: Principles and Practice of Sleep Medicine. Kryger MH, Roth T, Dement WC (Eds). WB Saunders Company, 1989, Cap. 11, p. 141-150.

2. Aschoff J. Circadian Rhythms in man. Science, 148:14271433, 1965.

3. Dinges DF. The influence of the human circadian timekeeping system on sleep. In: Principles and Practice of Sleep Medicine. Kryger MH, Roth T, Dement WC (Eds.) WB Saunders Company, 1989, Cap. 12, p. 153-162.

4. Giannela Neto D \& Minanni S. In: Wajchenberg BL (Ed.). Ritmos endócrinos. Tratado de Endocrínologia. Roca, Săo Paulo, 1992. cap. 3, p.71-82.

5. Smolensky MH \& D'Alonzo GE. Medical chronobiology: concepts and applications. Am Rev Respir Dis, 147:S2-S19, 1993.

6. Lothman EW. Functional anatomy: a challenge for the decade of the brain. Epilepsia, 32 (Suppl.5):S3-S13, 1991.

7. Langdon-Down \& Brain WR. Time of day in relation to convulsions in epilepsy. Lancet, i:1029-1932, 1929.

8. Patry FL. The relation of time of day, sleep and other factors to incidence of epileptic seizures. Am J Psychiatry, 87:789-
813,1931 . 
9. Milton JG, Gotman J, Remillard GM, Andermann F. Timing of seizure recurrence in epileptic patients: A statical analysis. Epilepsia, 28:471-478, 1987.

10. Temkin NR \& Davis GR. Stress as a risk factor for seizures among adults with epilepsy. Epilepsia, 25:450-456, 1984.

11. Poirel C \& Ennaji M. Circadian aspects of epileptic behavior in comparative psychophysiology. Psychol Rep, 68:783-801, 1991.

12. Stevens JR, Lonsbury BL, Goel SL. Seizure occurrence and interspike interval. Arch Neurol, 26:409-419, 1972.

13. Kellaway P, Frost JD, Crawley JW. Time modulation of spikeand-wave activity in generalized epilepsy. Ann Neurol, 8:491$500,1980$.

14. Lange HH, Lieb JP, Engel Jr J, Crandall PH. Temporo-spatial patterns of pre-ictal spike activity in human temporal lobe epilepsy. Electroencephalogr Clin Neurophysiol, 56:543-555, 1983.

15. Martins da Silva A, Aarts JHP, Binnie CD, Laxminarayan R, Lopes da Silva FH, Meijer JWA, Nagelkerk N. The circadian distribution of interictal epileptiform EEG activity. Electroencephalogr clin neurophyisiol, 58:1-13, 1984.

16. Kellaway $P$. Intensive monitoring in infants and children. Advances in neurology, 46:127-137, 1986.

17. Bowman T, Leppik I, Haus E. Periodicity of seizure activity in persons with complex partial seizures. Epilepsia, 25:658, 1984.

18. Poirel C. Circadian patterns of vigilance and seizure susceptibility in genetically epileptic mice: heuristic aspects in neurology. Advances in Chronobiology (Part B):459-466, 1987.

19. Gotman J. \& Koffler DJ. Interictal spiking increases after seizures but does not after decrease in medication. Electroencephalogr Clin neurophysiol, 72:7-15, 1989.

20. Balish M, Albert PS, Theodore WH. Seizure frequency in intractable partial epilepsy: a statistical analysis. Epilepsia, 32:642-649, 1991.

21. Nagao $H$, Morimoto $T$, Takahashi $M$, Habara $H$, Nagai $H$, Matsuda $\mathrm{H}$. The circadian rhythm of typical absence seizures - The frequency and duration of paroxysmal discharges. Neuropediatrics, 21:79-82, 1990.

22. Horita $H_{1}$ Uchida $E$, Maekawa $K$. Circadian rhythm of regular spike-wave discharges in childhood absence epilepsy. Brain Dev, 13:200-202, 1991.

23. Ellis CR. Chronobiological aspects of epileptic phenomena: a literature review, implications for nursing and suggestions for research. J Neuroscience Nursing, 24:335-339, 1992.

\section{Endereço para correspondência}

Universidade Federal de Sāo Paulo

Rua Pedro de Toledo, 655 - Vila Clementino

CEP 04039-030 - Sāo Paulo - SP

e-mail: cjcamp@ibm.net

ANAFRANIL*-Formas farmacêuticas e apresentaçōes: Drágeas com 10 ou $25 \mathrm{mg}$ de cloridrato de clomipramina; excipiente q.s.p. 1 drágea. Caixas com 20 drágeas. Ampolas com $25 \mathrm{mg}$ de cloridrato de clomipramina: veiculo q.s.p. $2 \mathrm{ml}$. Caixas com 10 ampoias. Anafranil SR: cada comprimido de liberaçảo lenta contem cloridrato de clomipramina $75 \mathrm{mg}$; excipiente q.s.p. 1 comprimido Caixas com 20 comprimidos. Posologia: O esquema posológico deve ser estabelecido individualmente. A dose diarıa usual è de $75-150 \mathrm{mg}$ (em ataques de pânico, 25 - $100 \mathrm{mg} / \mathrm{dia}$ ). Iniciar o tratamento com um comprimido de $25 \mathrm{mg}$, duas a trés vezes ao dia. ou com un comprimido de liberação lenta de $75 \mathrm{mg}$. uma vez ao dia; em ataques de pànıco. iniciar com um comprimido de $10 \mathrm{mg}$ por dia. Evitar a interrupção abrupta do tratame!to. Contra-indicaçōes: Hipersensibilidade à ciomipramina ou aos excipientes; sensibitidade cruzada aos antidepressivos triciclicos do grupo diberizazepına. Infarto do miocárdio recente. Uso concomitante cum inibidores da MAO. Precauçōes e advertências: Distúrbios da conduçao cardiaca, insuficiência cardiovascular, baixo timiar convulsivo, glaucoma de ângulo agudo, distúrbios da miç̧āo, insuficiència hepática grave. tumores da medula adrenal, terapia eletroconvulsiva. Hipertireoidismo ou tratamento concomitante com preparaçōes tireoidianas. Constipaçàu crónicd. Monitorizaçäo da contagem de células sangüineas e da funçāo hepatıca Intervençōes cirürgicas. Risco de suicidio. Choque anafilático (uso parenteral). Uso concomitante com drogas anti-hipertensivas. drogas simpatomiméticas. depressores do SNC, anticoliıérgicos, inibıdores seletivos da recaptaçào da serotonina, neurolèpticos. indutores das enzimas hepática, anticoagulantes, cimetidina, metilfenidato e estrogenos. Nào deve ser usado durante a gravidez e a lactação. Cuidado ao ope-rar máquinas e dirigir veiculos. Reaçōes adversas: Frequentes: reaçoes no SNC e reaçoes antıcolinergicas (sonolência, fadiga, tremor, mioclonia, aumento do apetite, boca seca. distúrbios da miç̧ao. distúrbios visuais). ganho de peso. Ocasionais: confusáo, alucinaçòes, agitação, distúrbios visuaıs. elevaçào das transaminases, reaçōes alérgicas cutáneas, disturbios gastrintestinais. Ruras: atıvaçào de sıntomas psicóticos, convulsỏes, ataxia, arrıtmıs. hıpertensào. Casos isolados: glaucoma. disturbios da conduçäo cardiaca, hepatıte. contagem anormal das células sangüineas. alveolite alérgica. reaçòes anafiláticasianafilactóides e hiperpırexia

Informaçōes completas para prescriçào à disposiçào da classe medica mediante solicitaçào.

TRILEPTAL - Apresentações: Caixas com 20 comprimidos divisiveis de 300 e $600 \mathrm{mg}$ de oxcarbazepina; frascos com $100 \mathrm{ml}$ de suspensão oral contendo oxcarbazepina a $6 \%$. Indicaçōes: Epilepsia (exceto ausência). Posologia: Aduftos: 300 a $3000 \mathrm{mg} / \mathrm{dia}$. Crianças: 10 a $30 \mathrm{mg} / \mathrm{kg} / \mathrm{dia}$. Administraçāo da dose diária em 3 ou 2 tomadas. Cada $\mathrm{ml}$ de Trileptal Suspensão contém $60 \mathrm{mg}$ de oxcarbazepina. Contra-indicaçōes: Hipersensibilidade à oxcarbazepina. Bloqueio atrioventricular.Precauções/Cuidados: Gravidez e lactação. Monitorização dos níveis séricos de sódio. Disfunção renal grave. Interrup̧̧āo abrupta do tratamento. Alergia cruzada à carbamazepina. Álcool. Contraceptivos orais. Cuidado ao dirigir ou ao operar máquinas. Reações adversas: Reaçōes adversas moderadas, a maioria transitórias. Freqüentes: fadiga. Ocasionais: sonolência, decréscimo na contagem de leucócitos (oscilante, transitório), hiponatremia, conduzindo em casos raros a intoxicação aquosa. Raras: instabilidade psíquica, depressão, trombocitopenia, pancitopenia. Casos isolados: Sindrome de Stevens-Johnson.

Informações completas para prescrição à disposição da classe médica mediante solicitação. 